\title{
An integrated system for a real time fight against maritime and coastal pollution with environmental data acquisition, pollutant spillages modelling and risk management
}

\author{
G. Winter, B. Galván, J. P. Ramos, H. Carmona, B. González, \\ J. I. Jiménez, A. Cacereño \& S. Alonso \\ Lab CEANI, Institute of Intelligent Systems and Numerical Applications \\ in Engineering (IUSIANI), University of Las Palmas de Gran Canaria, \\ Spain
}

\begin{abstract}
A new integrated system with the capacity to help minimize the coastal environmental impacts when a pollutant load is discharged into the sea, in particular oil spills, is presented. The system, named SAMM, integrates simulation models (oil spills, dumping of sewage, brine, etc.) and risk assessment tools for decision-making in emergency situations so as to minimize the environmental and socio-economic impact of marine spills. The main core modules of the integrated system are: Monitoring and Data, Simulations and Risk Analysis. The present system has been developed by an interdisciplinary team that has worked on many previous projects in the field and recently received a national award. This paper describes the modelling implemented in the integrated system and shows examples of real applications in coastal areas and seas.
\end{abstract}

Keywords: management of water pollution, environmental monitoring, oil spill.

\section{Introduction}

The SAMM system integrates simulation models (oil spills, dumping of sewage, brine, etc.) and risk assessment tools for decision-making in emergency situations so as to minimize the environmental and socio-economic impact of marine spills. 
The system is open in that it has interfaces for real time capture of data of a varied nature without being dependent on any of them. It is flexible, with its modular nature enabling it to adapt to requirements as they vary over time. The inclusion in the system of new types of incidents, new risk scenarios and situations or a geographical relocation of the analysis environment are some of the features worthy of mention. An interesting additional feature available with the system is that of allowing the operator to simulate a future hypothetical situation based on the currently monitored situation or on other hypothetical situations. This concept is of crucial importance when we speak of early alerts.

The main core modules of the integrated system are:

- Monitoring and Data: On-line data (wind, sea current, wave height, temperature, etc.) are automatically retrieved (in real time) from many different sources or manually inserted by users. The Real Time Monitoring System (RTMS) allows the detection of potentially dangerous scenarios that could give rise to accidental marine pollution and to activate early alert calls based on a computed risk level.

- Simulations: Giving access to a complete set of tools for oil spill simulations management (creation, edition, modifying, etc., with different spill models). Many different simulations can be performed at the same time, so the influence of the different decisions and/or changes in the environmental situation can be compared in terms of water pollution or coastal impact.

- Risk Analysis: Which provides a list of risk scenarios generated in accordance with the Data, the results of the RTMS, the Geographic Information System (GIS) and an expert's rule based system. When simulations are carried out, the evolution of the risk level through every simulation is independently computed and showed too.

\section{Functional tools of the SAMM system}

Noteworthy among the functionalities of SAMM are:

- Real time data capture. - Dedicated to the insertion into the system in real time of data related to external events such as buoy data (winds, currents, etc.), maritime traffic and others.

- Integrated graphic interface. - Dedicated to graphic representation, in user selected layers, of all the information available to the system: buoys and relevant data (Winds, Currents, Wave Height, Temperature), Vessels (course and load), in addition to data manually introduced by the user, Alert levels, and Spill Simulations. The integrated graphic interface is highly configurable. The operator can hide the non-relevant information or position the display windows as he or she sees fit. As it is a multi-window system with multi-monitor support, the operator can distribute the windows among the various monitors as required.

- Geographical representation. - Used to locate in a specific geographical environment the information received and/or processed by the system. A zoom is available for specified geographical areas. The presented data are classified in layers to facilitate operator navigation. The information as it is 
presented on the screen is synchronised at any given moment in all the different possible display views in which it appears. The system displays the information in multiple views.

- Risk analysis, alert system and response protocol system. - A set of rulemaking decisions enables analysis of existing risks, establishment of alert levels and ordering of response protocols. The rules are established through Probabilistic Analysis of Risks and Event Trees that can be associated with the different situations (scenarios) that are possible in the environment. The rules can be modified, complemented, or replaced with others that come from previous experiences and/or subject matter experts. The risk viewer represents the alert level for the current visible situation and the different risk scenarios inspected by the system for this situation.

- Hydrocarbon spill simulation. - The system uses programmes that enable drift prediction after an involuntary and/or accidental oil spill. Spill environment characteristics (Winds, Currents, Waves and Temperature) are taken into account in the simulations, as well as the physical/chemical characteristics of the hydrocarbons involved. The temporal evolution of the physical/chemical characteristics of the hydrocarbon while it is in the sea (weathering) can be determined, as well as when it reaches land should such a circumstance occur. The spill simulations are carried out using the system's Integrated Graphic Interface.

- Forecast of coastal impact and damage. - The combined action of the Integrated Graphic Interface, GIS and Spill Simulation allows forecasts to be made of the areas where the spill will pass by and impact with the coast. Evaluation of coastal impact and weathering enables a prediction of the extent of coastal damage.

- Integrated historical archive. - At any given moment the operator has available an observation time framework. He or she can quickly consult a past situation or hypothetical future situations that have been introduced.

- Early alert system. - Responsible for performing the following controls for the area under observance:

- Control of maritime traffic.

- Control of weather conditions.

- Control of protected areas (distances).

- Predefined Alert Rules.

- Automatic valuation of Alert Rules.

- Current alert level display (number/colour).

- Risk Management System. - Performs the following functions:

- Scenario capture for simulation of alternatives.

- Listing of situations that give rise to protocols.

- Personalised response protocols in accordance with emergencies definable/modifiable by the user.

- Incorporation of predefined protocols.

- Simulations. - The system allows the editing of risk situations and corresponding data, enabling it to create simulations in the event of hypothetical events related to hydrocarbon spills at sea. 


\section{Structure of the SAMM system}

The overall design of the system allows the incorporation of the latest advances in software development, at both scaleable and dynamic levels, enabling the progressive incorporation of new software components based on their availability and without the need for the whole programme to be fully functional. The diagram below shows the structure of the system:

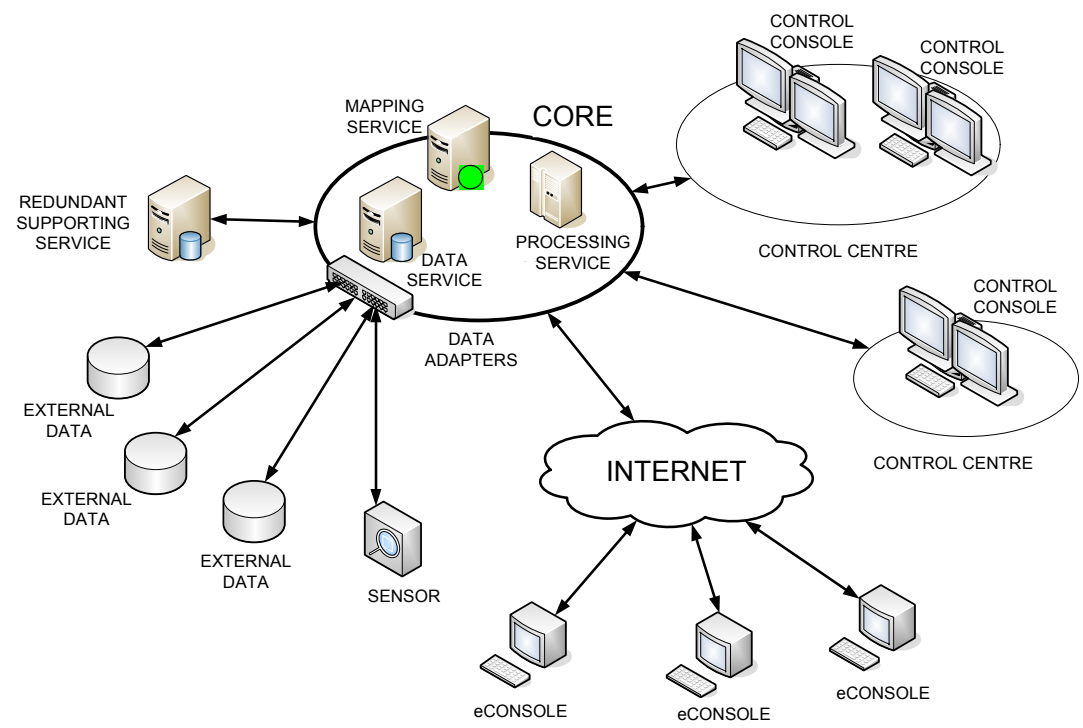

Figure 1: Structure of the SAMM system.

The core is basically responsible for coordinating/distributing/managing the available processes, data and resources, controlling the interaction with the databases and supervising the global connectivity, integrity and security of the system. Additionally, it provides the following essential services: Information Processing Service, Data Service and Mapping Service. State-of-the-art technology has been chosen to meet these requirements including, amongst others, the Microsoft .Net Framework 3.0 platform, with support for Windows Vista, 64 bits and multithreading.

A processing management system has been designed and implemented which enables encapsulation of the different operations which modify the status of the system to allow their serialisation and later transfer between the different consoles and system server, providing a high level technological platform that guarantees synchronisation of the different SAMM terminals. This system enables centralised processing of the operations performed, which facilitates the development of recovery methods in the event of failures, system status monitoring and permission management. This processing management system has been designed in such a way that the definition of a specific operation is 
uncoupled from its final implementation, enabling the different packets to establish new implementations tailored to their field of action.

Notable among the main design features of SAMM are its flexible and open nature, its sophisticated user-friendly interface, and the use of the latest available state-of-the-art technology in its development.

\section{Utilities and applications}

SAMM is a tool for the creation and edition of simulations (hypothetical situations in the monitoring zone) so that users can define any meteorological or maritime traffic condition. The monitored real information can be used initially in the simulations and afterwards be modified and adapted. This way, SAMM is a tool that allows evaluating possible evolutions from the current situation and pondering the impact that different actions can produce on the system level of alert. It also permits to study past situations and weight the alternatives that could have been taken to improve the response in similar situations.

SAMM provides oil and other pollutants spill simulations tools able of simulating sceneries with different meteorological conditions, of seeing how the spill with behave, or obtaining physic-chemistry information about the spill in every point of time and space of the simulation taking into account its degradation and climate conditions. It also can determine the impact of the spill in the coast and its behaviour. It is also possible to compare several simulations made with different parameters or models of simulations. On the other hand, it allows doing on-line simulations from other remote stations, so that it gives access and the necessary power of calculus to do simulations to other users. The system can be used as a test bed for the research and development of new algorithms and heuristics for risk management, oil spill simulations and other spills.

SAMM can give a remote test bed to help researchers to develop new modules using the power of the system, enhancing the cooperative research and the study of environmental prevention techniques. In this way, SAMM already incorporates different programmes to simulate marine currents [4], dumping of sewage and brine in offshore waters $[5,6]$ and oil slick path, weathering and oil slick-coast interaction [1].

A 3D mass consistent model with finite volumes for marine current simulation was implemented. Basically, this programme looks for a vector field $\mathbf{u}$ that approaches another vector field $\mathbf{u}_{0}$ that is the result of interpolating experimental measurements of marine current velocities obtained from current meters. The new vector field $\mathbf{u}$ has to verify, on the one hand, the mass conservation law, on the other hand, the velocity field deviation by the marine bottom orography. The problem to solve is the following one:

$$
\begin{array}{ll}
\operatorname{div} \mathbf{u}=0 & \text { in the domain of study } \\
\mathbf{u} \cdot \mathbf{n}=0 & \text { on the marine bottom } \\
\frac{\partial \mathbf{u}}{\partial \mathbf{n}}=0 & \text { on the rest of the boundaries }
\end{array}
$$


In order to simulate the dumping of sewage and brine in offshore waters, a 3D Taylor-Galerkin version with finite volumes for the pollutant concentration simulation was implemented. The dynamic mathematical expression for the concentration of waste $c$ for three dimensions that includes the effect of diffusion and degradation may be expressed as:

$$
\frac{\partial c}{\partial t}+\frac{\partial(u c)}{\partial x}+\frac{\partial(v c)}{\partial y}+\frac{\partial(w c)}{\partial z}-\frac{\partial}{\partial x}\left(D_{x} \frac{\partial c}{\partial x}\right)-\frac{\partial}{\partial y}\left(D_{y} \frac{\partial c}{\partial y}\right)-\frac{\partial}{\partial z}\left(D_{z} \frac{\partial c}{\partial z}\right)+k c-S_{S}=0
$$

where $c$ is the mean concentration averaged over a time much larger than the typical time scale of turbulent fluctuations; $u, v$ and $w$ are respective components of the velocity $\mathbf{u} ; D_{x}, D_{y}$ and $D_{z}$ are the respective components of the diffusion diagonal tensor $\mathbf{D} ; k$ is the decay coefficient for non-conservative substances (e.g. $k=(5.10-7-10-5) \mathrm{s}^{-1}$ for coliform concentration); and $S_{S}$ is the all important source terms.

A numerical scheme assessment and a study of the unsteady problem accuracy have been carried out in [5]. The results obtained with the model validation test had very satisfactory, since the approximated solution obtained with the model for the pollutant concentration calculation was very similar to the analytical solution. Furthermore, the model does not suffer from false diffusion in multi-dimensional flows although the velocity vector is not parallel to one of the coordinate directions.

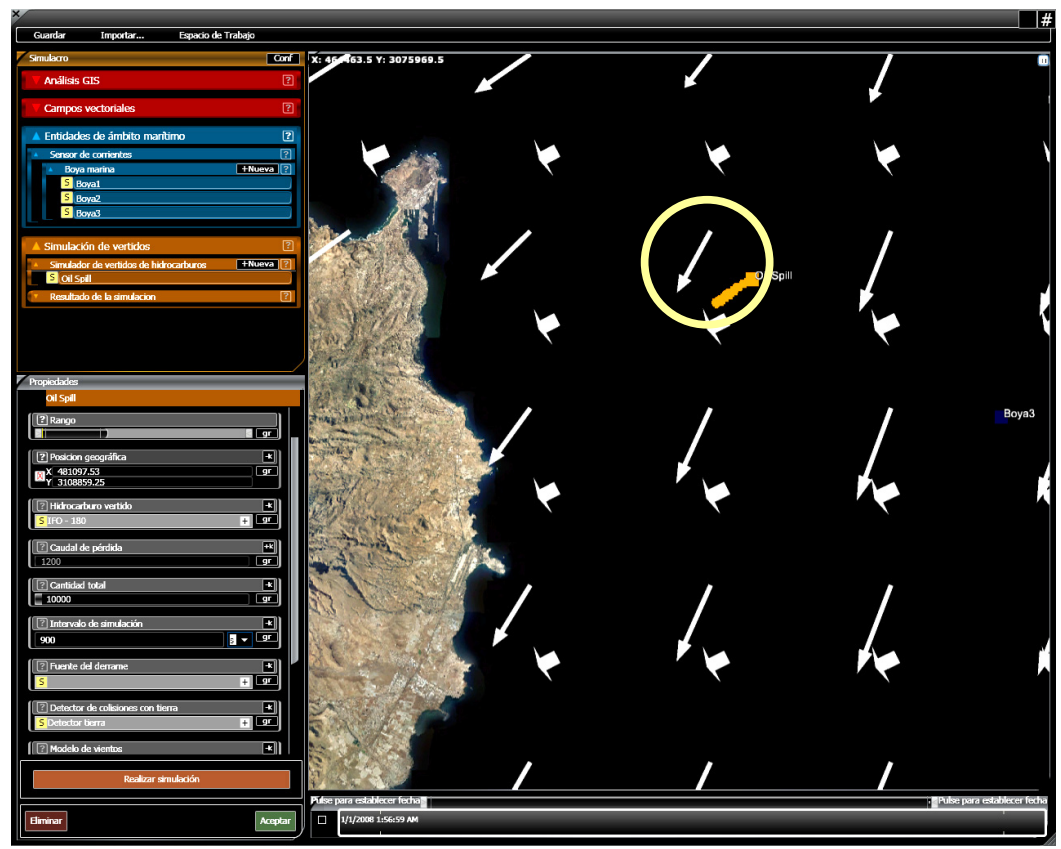

Figure 2: Wind (big arrows) and marine current (small arrows) fields are shown with an oil slick that is going from NE to SW (see the circle). 


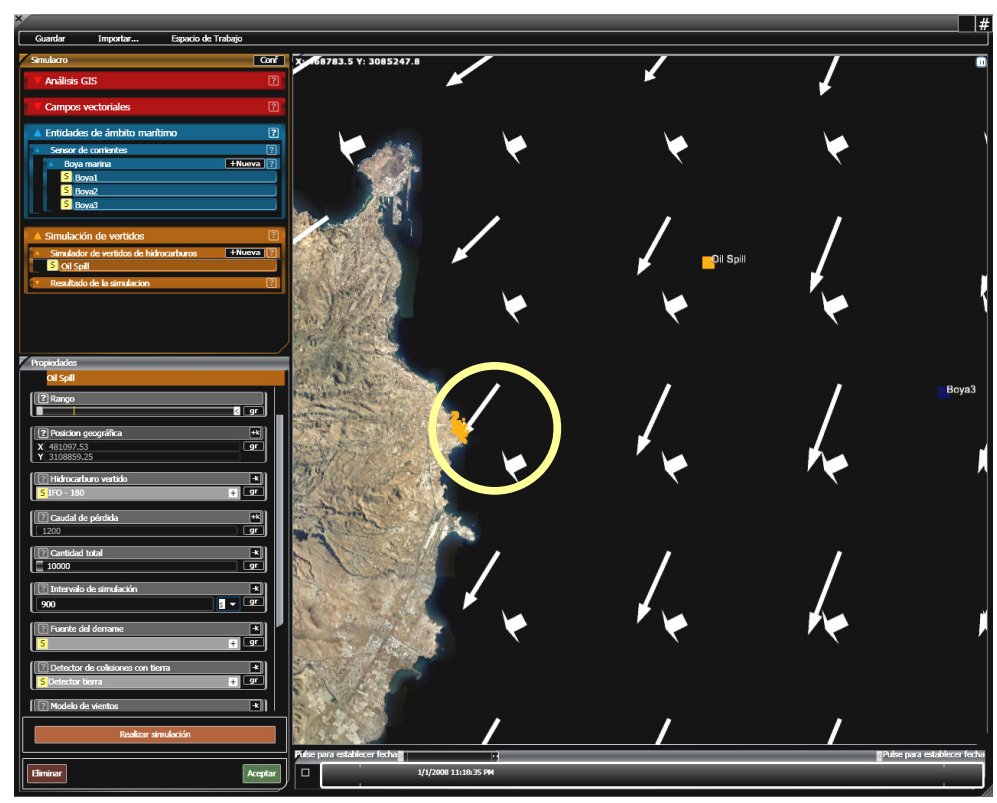

Figure 3: Wind (big arrows) and marine current (small arrows) fields are shown with an oil slick that is hitting the coast (see the circle).

With respect to the oil slick path, a Lagrangian model was implemented, in which a drift angle for the slick has been incorporated (between $0^{\circ}$ and $13^{\circ}$ in accordance with field and laboratory experiments and previous oil spill data [7]) which is randomly generated for each time step in the execution of the software. As for the spreading of the oil slick, random speeds are determined with a uniform distribution proportional to the diffusion coefficients provided by Fay's formula [8] - with the constant $\mathrm{k} 2=0.725$ (value recommended by Flores et al. [9]) - and the relation between the coefficients of diffusion and the fluctuation intervals of the speeds given by Leitão [10]. The random speeds are determined as proposed by Proctor et al. [11].

With regards to the question of weathering, new methodology from Fingas $[12,13]$ to evaluate the rate of evaporation and the quantitative prediction of emulsification in the slick has been incorporated.

Finally, the length of the shoreline contaminated by oil slicks transported in the nearshore environment is evaluated following the work from Cheng et al. [14].

The screen shot shown in fig. 4 is related to external data capture by the application (in this case of winds from buoys or weather stations), and to the mesh generated for interpolation. As can be seen, the user defines the mesh in accordance with the required resolution. Two different meshes can be seen from wind velocity data obtained at different weather stations. The larger scale shows data at ocean level, while the smaller scale shows a detail at port level where a higher resolution is required. 


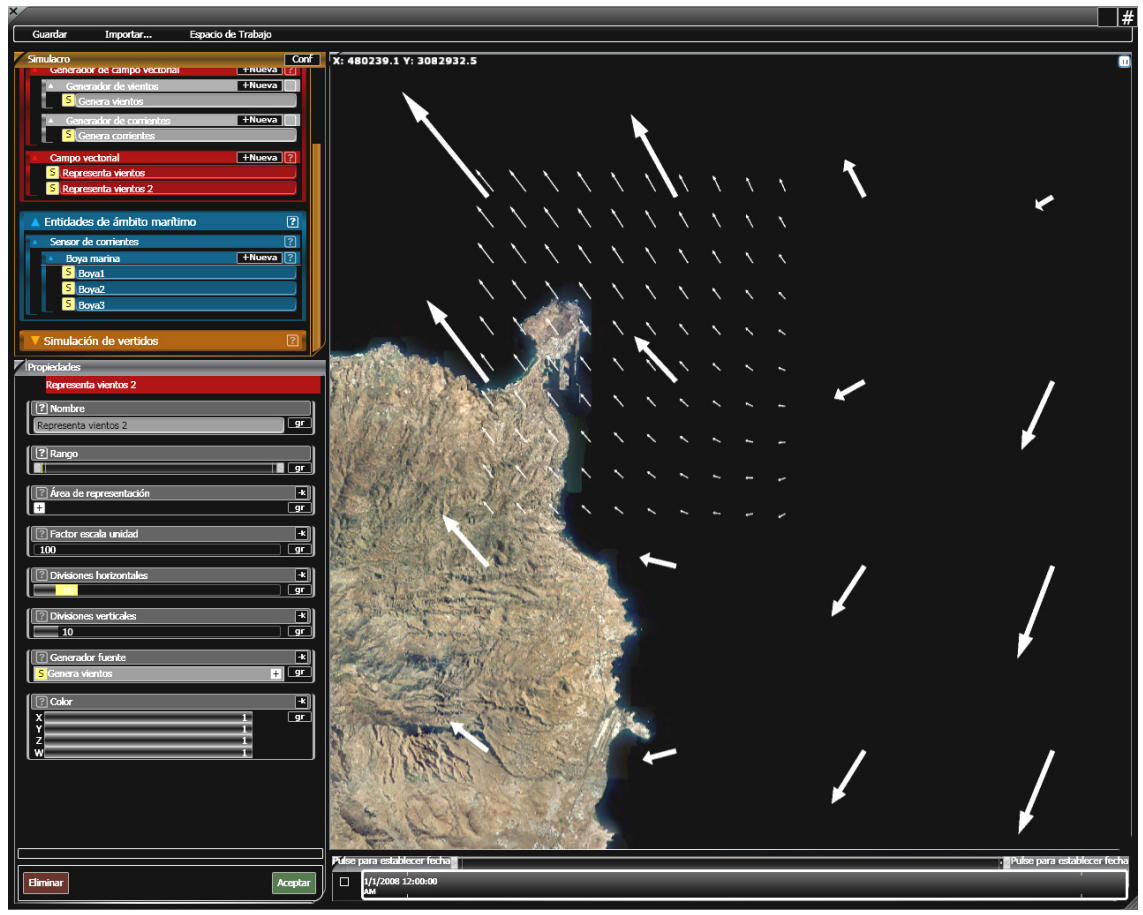

Figure 4: Screen shot related to external data capture by the application (in this case of winds from buoys or weather stations), and to the mesh generated for interpolation.

\section{References}

[1] González, B., Galván, B. Benítez, E., Martín, I., Ramos, J.P., Carmona, H. \& Winter, G. Robust optimisation of coastal environmental impact from oil spills using a flexible evolution agent and finding the optimal course of the damaged tanker. Water Pollution VIII. Modelling, Monitoring and Management. (Series: WIT Transactions on Ecology and the Environment), WIT Press, 95, pp. 263-272, 2006.

[2] Integrated system for risk management, environmental monitoring and fight against the maritime and coastal pollution in real time and remote access. Project of Technical Research Promotion (PROFIT), CIT-310100-2007-13, supported by Ministry of Education and Science, Spain, 2007-2008.

[3] Winter, G. and Galván, B. Desarrollo y aplicación de modelos y sistemas integrados ALERMAC y SAM para la predicción del comportamiento de derrames superficiales de hidrocarburos en el medio marino y de los procesos de transformación del vertido. Serie Investigación del Ministerio de la Presidencia, Secretaría General Técnica, Imprenta Nacional del B.O.E. Catálogo general de publicaciones oficiales: http://0.60.es. 
[4] González, B. and Winter, G. Optimising the Determination of Multiple Waste Disposal Sites in Offshore Waters Using Genetic Algorithms. Proc. of the European Congress on Computational Methods in Applied Sciences and Engineering (ECCOMAS 2000), Barcelona, 2000.

[5] González, B. and Winter, G. A 3D finite volume model of the pollutant dispersion in offshore waters. Proc. of the Adaptive Modeling and Simulation (ADMOS 2003), an IACM Special Interest Conference and an ECCOMAS Thematic, Chalmers University of Technology, 2003.

[6] González, B., Galván, B. \& Winter G. Economic and environmental optimal control in dumping of sewage. Coastal Engineering VI. Computer Modelling and Experimental Measurements of Seas and Coastal Regions. (Series: Environmental Studies). C.A. Brebbia, D. Almorza \& F. LópezAguayo (Eds.), 9, pp. 143-152, 2003.

[7] Stolzenbach, K.D., Madsen, O.S., Adams, E.E., Pollack, A.M., Cooper, C.K. A review and evaluation of basic techniques for predicting the behavior of surface oil slicks, Report No. 22, MIT: Cambridge, Mass. USA, 1977.

[8] Fay, J.A. The spread of oil slicks on a calm sea. Fluid Mechanics Laboratory, Dept. of Mech. Eng., MIT: Cambridge, MA, USA, 1969.

[9] Flores, H., Andreatta, A., Llona, G. and Saavedra, I., Measurements of oil spill spreading in a wave tank using digital image processing. Oil and hydrocarbon spills, modeling, analysis and control, WIT Press, Southampton, UK, pp. 165-173, 1998.

[10] Leitão, P., Modelo de dispersão lagrangeano tridimensional, dissertaçao de mestrado, Instituto Superior Técnico, Universidade Técnica de Lisboa, Lisboa, 1996.

[11] Proctor, R., Flather, R.A., Elliot, A.J., Modelling tides and surface drift in the Arabian Gulf, application to de Gulf oil spill. Continental Shelf Res 1, pp. 531-545, 1994.

[12] Fingas, M.F., Modeling evaporation using models that are not boundarylayer regulated, Journal of Hazardous Materials, 107, pp 27-36, 2004.

[13] Fingas, M.F. and Fieldhouse, B., Formation of water-in-oil emulsions and application to oil spill modelling, Journal of Hazardous Materials, 107, pp 37-50, 2004.

[14] Cheng, N.-S., Law, A. W.-K., Findikakis, A.N., Oil Transport in Surf Zone, Journal of Hydraulic Engineering, 126 (11), pp. 803-809, 2000. 